\title{
Comparação da destreza manual em indivíduos com lateralidade distinta
}

\author{
Comparison of manual dexterity in individuals with distinct laterality
}

\author{
Lidiane Aparecida Fernandes ${ }^{1}$, Wallace Tadeu Alves², Simara Regina de Oliveira Ribeiro ${ }^{3}$, \\ Lucas Savassi Figueiredo ${ }^{3}$, Natália Fontes Alves Ambrósio ${ }^{4}$, Crislaine Rangel Couto ${ }^{5}$, Guilherme Menezes Lage ${ }^{3}$ \\ ${ }^{1}$ Universidade Federal de Juiz de Fora (UFJF-GV), Governador Valadares, Brasil \\ 2 Faculdade de Ensino de Minas Gerais (FACEMG), Belo Horizonte, Brasil \\ ${ }^{3}$ Universidade Federal de Minas Gerais (UFMG), Belo Horizonte, Brasil \\ ${ }^{4}$ Centro Universitário Una (UNA), Belo Horizonte, Brasil \\ ${ }^{5}$ Centro Universitário Metodista Izabela Hendrix (CEUMIH), Belo Horizonte, Brasil
}

\section{HISTÓRICO DO ARTIGO}

Recebido: 28 janeiro 2021

Revisado: 22 abril 2021

Aprovado: 24 abril 2021

\section{PALAVRAS-CHAVE:}

Destreza manual; Lateralidade; Controle motor.

\section{KEYWORDS:}

Manual dexterity; Laterality; Motor control.

\section{RESUMO}

INTRODUÇÃO: A destreza manual pode ser definida como a habilidade e a facilidade na utilização das mãos, como escrever, dedilhar um instrumento musical, lançar e apanhar. A lateralidade pode ser conceituada como a preferência de utilização por um dos membros em detrimento ao outro e se confirma, por exemplo, em indivíduos destros e canhotos, influenciando nos distintos desempenhos das mãos.

OBJETIVO: Comparar a destreza manual em destros e canhotos através da tarefa de colocar e retirar pinos no Grooved Pegboard Test.

MÉTODOS: A amostra foi composta por 20 voluntários saudáveis, de ambos os sexos, com idade entre 18 e 40 anos, dividida em dois grupos, sendo: 5 homens e 5 mulheres de população destra e 5 homens e 5 mulheres de população canhota. Referente ao índice de dominância lateral, os indivíduos destros apresentaram índice médio de $97 \%$ e os indivíduos canhotos de $91 \%$ no Inventário de Dominância Lateral de Edimburgo.

RESULTADOS: Na condição de colocar os pinos foram observadas diferenças entre as mãos dos destros $[t(d f=9)=-7,16 ; p=0,01]$ e dos canhotos $[t(d f=9)=4,80 ; p=0,01]$, o uso da mão preferida apresentou melhor desempenho relacionado ao uso da mão não preferida. Já para a condição de retirar os pinos não foram observadas diferenças entre as mãos direita e esquerda para os destros $[t(d f=9)=-1,96 ; p=0,08]$ e nem para os canhotos $[t(d f=9)=1,27 ; p=0,23]$. Em relação ao índice de assimetria não foram observadas diferenças entre os grupos, apesar de parecer que as assimetrias se destacam em tarefas nas quais a demanda cognitiva é maior do que a demanda energética.

CONCLUSÃO: O desempenho nas tarefas de destreza manual foi diferente para a mão preferida tanto para destros quanto para canhotos, sendo que a mão preferida apresentou melhor desempenho quando comparada à mão não preferida.

\section{ABSTRACT}

BACKGROUND: A manual dexterity can be defined as the skill and ease of using the hands, such as writing, strumming a musical instrument, throwing and catching. Laterality can be conceptualized as the preference of use by one of the members over the other and it is confirmed, for example, in right-handed and left-handed individuals, influencing the different performances of the hands.

OBJECTIVE: To compare manual dexterity in right-handed and left-handed users by placing and removing pins in the Grooved Pegboard Test.

METHODS: The sample consisted of 20 healthy volunteers, of both sexes, aged between 18 and 40 years, divided into two groups: 5 men and 5 women from the right population and 5 men and 5 women from the population left-handed. Regarding the lateral dominance index, right-handed individuals had an average index of $97 \%$ and left-handed individuals, $91 \%$, in the Edinburgh Lateral Dominance Inventory.

RESULTS: In the condition of placing the pins, differences were observed between the right-handed $[t(d f=9)=-$ 7.16; $p=0.01]$ and left-handed $[t(d f=9)=4.80 ; p=0.01]$, where the use of the preferred hand showed better performance related to the use of the non-preferred hand. As for the condition of removing the pins, no difference was observed between the right and left hands for the right-handed $[t(d f=9)=-1.96 ; p=0.08]$ and neither for the left-handed $[t(d f=9)=1.27 ; p=0.23]$. Regarding the asymmetry index, no differences were observed between the groups, although it seems that the asymmetries stand out in tasks where the cognitive demand is greater than the energy demand.

CONCLUSION: The performance in the tasks of manual dexterity was different for the preferred hand for both right-handed and left-handed people, with the preferred hand presenting a better performance when compared to the non-preferred hand. 


\section{INTRODUÇÃo}

A destreza manual pode ser definida como a habilidade e a facilidade na utilização das mãos, e é subdivida em duas classes: fina e global (DESROSIERS, 1997; VAN DE VEN-STEVENS et al., 2009). A destreza manual fina se caracteriza pela habilidade de manipular pequenos objetos utilizando os dedos das mãos, por meio de movimentos de pinças, tais como: pinça polpa a polpa, pinça lateral ou pinça trípode. A destreza manual global, consiste em manipular grandes objetos, de forma coordenada com o uso das mãos e dos dedos.

De forma geral, a destreza manual permite que o indivíduo realize suas atividades diárias com ambas as mãos coordenadamente, mão preferida e mão não preferida (DESROSIERS, 1997). O desenvolvimento da destreza manual está associado com a maturação do trato corticoespinhal, o que influencia na escoIha da mão dominante (FUELSCHER et al., 2021). Além disso, os fatores ambientais e biológicos podem influenciar a destreza manual, como exemplo, o sexo, as características culturais e a lateralidade.

A capacidade de reproduzir resultados planejados com êxito é fortemente influenciada pela lateralidade que representa um importante fator na motricidade humana (SERAFIM et al., 2000; FERNANDES et al., 2020). A lateralidade pode ser conceituada como a preferência de utilização de um dos membros em detrimento do outro e se confirma, por exemplo, na preferência manual, vista através da utilização de uma mão em relação à outra (PORAC; COREN, 1981; TEIXEIRA, 2006). De acordo com Souza e Teixeira (2011) "a lateralidade é compreendida como um elemento dinâmico da motricidade humana, em que predisposições inatas são reforçadas ou modificadas pela contínua interação com o ambiente durante o ciclo de vida de um indivíduo".

Estudos como os de Salvador et al. (2017) e Fernandes et al. (2018) buscaram comparar o desempenho das mãos em diferentes tarefas. Os resultados desses estudos convergem para o entendimento de que em destros a mão preferida é frequentemente mais rápida e mais precisa do que a mão não preferida. De acordo com Boulinguez et al., (2000) a mão não preferida tende a apresentar menor tempo de reação, reforçando novamente as diferenças de desempenho entre as mãos. A diferença no desempenho das mãos nas mais diversas tarefas é conferida a uma proficiência relativa dos hemisférios cerebrais no processamento de informações percepto-motoras (TODOR; SMILEY, 1985).

Tzourio-Mazoyer et al. (2021) investigaram a ativação cerebelar envolvida na preferência manual de destros e canhotos e identificaram ativações contralaterais e desativações ipsilaterais, com maiores assimetrias quando se utilizou a mão preferida. Além disso, os autores identificaram dois diferentes mecanismos que permitem explicar a distribuição da lateralidade. 0 primeiro mecanismo aponta para atividade contralateral mais forte das áreas motoras corticais e cerebelares durante o movimento da mão direita, observada em canhotos e destros, mas modulada pela lateralidade. O segundo mecanismo evidencia uma desativação mais forte nas áreas ipsilaterais durante o movimento da mão não dominante em ambos os grupos (destros e canhotos).

Os estudos como o de Bagi et al. (2011) e Steenhuis et al. (1999) mostraram que os indivíduos canhotos são menos latera- lizados quando comparados aos destros, apresentando melhor desempenho com a mão preferida, esquerda, como exemplo, em tarefas que envolvem destreza manual fina (McGRATH; KANTAK, 2015). Para Judge e Stirling (2003) no processo de adaptação cultural os canhotos acabam se tornando mais proficientes com a mão não preferida em relação aos destros, o que Ihes garantiria durante a execução de tarefas que combinem os dois lados do corpo uma menor diferença de desempenho entre os membros contralaterais (GORYNIA; EGENTER, 2000).

Em contrapartida, para Giagazoglu et al. (2001), os indivíduos destros apresentam uma melhor coordenação motora em relação a indivíduos canhotos para tarefas manuais. Apesar de não representar o único fator que influencia na definição da lateralidade, a prática parece desempenhar um papel relevante na formação e consolidação de assimetrias neurais e comportamentais (MARCONI; MONTEIRO; OKAZAKI, 2019).

Os indivíduos canhotos tendem a ser menos lateralizados, o que permite especular que são menos assimétricos quando comparados aos destros. A assimetria de desempenho pode ser caracterizada como a diferença no desempenho do movimento entre as mãos (WOODWORTH, 1899), uma vez que os canhotos possuem um maior equilíbrio na utilização de ambas as mãos, pode-se esperar que a diferença no desempenho das mãos preferida e não preferida seja significativamente menor quando comparada à diferença do desempenho dos destros.

As diferenças no controle dos movimentos manuais entre indivíduos com lateralidade distinta podem ser explicadas a partir de uma análise dos fatores genéticos. Para Goodale (1990) os genes trariam consigo a informação para o desenvolvimento no hemisfério cerebral, determinando qual hemisfério será predominante para cada função. Dessa forma, em pessoas destras caberá ao hemisfério cerebral direito a responsabilidade pelo processamento paralelo e a percepção de aspectos relacionados ao ambiente, já o hemisfério esquerdo desempenha o papel de sequenciamento e temporização dos movimentos.

A literatura ainda apresenta a hipótese "right-based world" ou mundo orientado à direita (PORAC; COREN, 1981) para explicar a dominância manual. Nessa perspectiva, é postulado que para indivíduos canhotos é necessária uma adaptação ao ambiente físico, sendo compelidos a utilizar utensílios que na maioria das vezes não são adaptados a canhotos como tesouras, mesas etc. As características do ambiente físico e utensílios forçariam a utilização da mão não preferida por indivíduos dessa população, fato decorrente de uma predominância populacional de indivíduos destros (GURD et al., 2006).

Portanto, os indivíduos com lateralidade distinta possivelmente apresentam diferenças no desempenho de uma tarefa de destreza manual, entretanto, a literatura é escassa e muitas vezes contraditória na apresentação dessa relação (PRZYBYLA et al., 2012). Mais estudos são necessários para possibilitar a compreensão das diferenças no controle motor de indivíduos destros e canhotos. O entendimento sobre as diferenças no controle entre essas duas populações permite respaldar a intervenção profissional no âmbito do treinamento esportivo e da reabilitação motora.

O objetivo do presente estudo foi comparar a destreza manual em indivíduos com lateralidade distinta, tendo como campo de investigação o desempenho em indivíduos destros e canhotos, através da análise de desempenho na tarefa de colocar e retirar pinos no Grooved Pegboard Test. 


\section{MÉTODOS}

A amostra foi composta por 20 voluntários, saudáveis, de ambos os sexos, com idade entre 18 e 40 anos (média de $27 \pm 7,3$ anos). Os voluntários foram divididos em um grupo de destros ( 5 homens e 5 mulheres) e um grupo de canhotos ( 5 homens e 5 mulheres). Como critério de inclusão, os voluntários deveriam apresentar o Índice de Lateralidade superior a 80 pontos no Inventário de Dominância Lateral de Edimburgo (OLDFIELD, 1971).

Foram excluídos da amostra os voluntários que possuíam habilidades musicais. Para as voluntárias do sexo feminino, foram excluídas da amostra aquelas que apresentaram unhas compridas, unhas postiças ou outros de mesma natureza, para evitar a interferência no tempo de execução do movimento.

Os voluntários assinaram o Termo de Consentimento Livre e Esclarecido (TCLE). O estudo foi aprovado pelo Comitê de Ética em Pesquisa da Universidade Federal de Minas Gerais (CAAE 02437218.3.0000.5149), sob o Parecer N³082606.

Para a determinação do índice de lateralidade, foi utilizado o Inventário de Dominância Lateral de Edimburgo (OLDFIELD, 1971), constituído por 10 questões sobre preferência lateral na execução de tarefas realizadas no cotidiano. Para cada item assinalado foi computado um ponto, cuja somatória foi utilizada no cálculo do Índice de Preferência Lateral (TEIXEIRA; PAROLI, 2000). Para a realização do teste de destreza manual, o apareIho utilizado foi o Grooved Pegboard Test (Lafayette Instrument Company, modelo no 32025).

O Grooved Pegboard Test é composto por uma superfície com 25 orifícios, cada um possuindo uma reentrância posicionada em diferentes direções, e uma superfície côncava onde são colocados os pinos (FIGURA 1). Os pinos também possuem uma reentrância, que deve coincidir espacialmente com a do orifício para que haja encaixe (SALVADOR et al., 2017; FERNANDES et al. 2018)

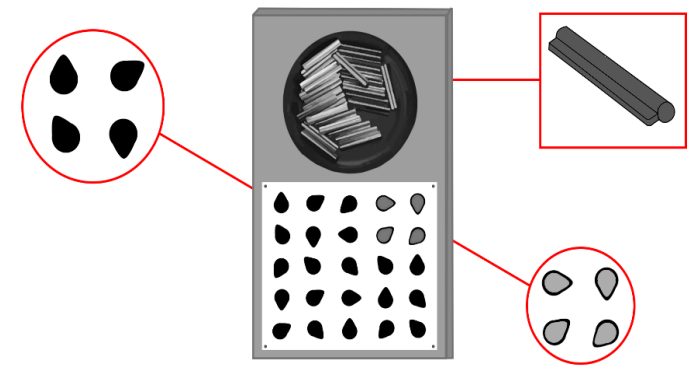

Figura 1. Ilustração do aparelho Grooved Pegboard Test e da orientação dos orifícios, cada um possuindo uma reentrância posicionada em diferentes direções.

Inicialmente os voluntários deveriam encaixar os 25 pinos em um receptáculo, um por vez, o mais rápido possível, na ordem prescrita. Conforme realizado nos estudos de Salvador et al. (2017) e Fernandes et al. (2018), durante a realização da tarefa com a mão direita, o preenchimento dos receptáculos ocorreu da esquerda para a direita, de cima para baixo, já o movimento realizado com a mão esquerda no sentido inverso, da direita para a esquerda, de cima para baixo.

O segundo momento da tarefa consistiu na retirada dos 25 pinos que foram encaixados nos orifícios seguida pela devolução ao receptáculo um a cada vez, o mais rápido possível, na ordem e direção prescritas (Figura 2a e 2b). Para a mão direita a retirada deveria acontecer da esquerda para a direita de baixo para cima e para a mão esquerda no sentido inverso, da direita para a esquerda de baixo para cima (BRYDEN; ROY, 2005). A ordem na qual a mão iniciou a tarefa foi contrabalançada entre participantes para eliminar qualquer efeito da ordem de execução no desempenho motor. a

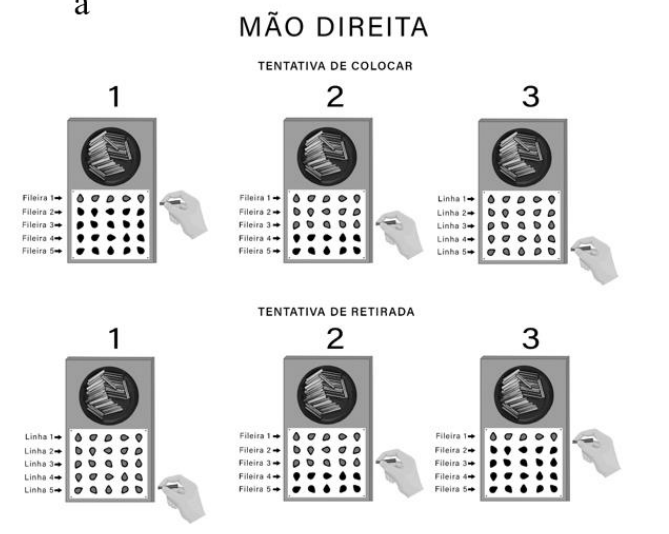

b

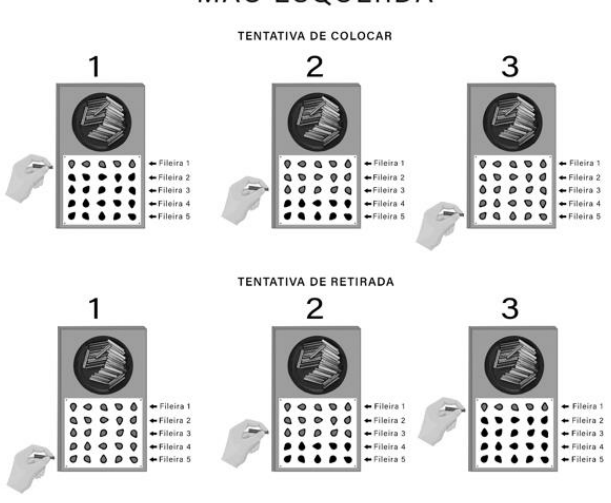

Figura 2. Tarefa de avaliação da destreza manual Grooved Pegobard Test e a ordem prescrita de execução da tarefa para a mão direita (a) e esquerda (b).

Obs: Os números representam as fases da tentativa de colocar o pino, sendo:

$1=$ início da tentativa para a mão direita

$2=$ meio da tentativa de colocar para a mão direita

3 = final da tentativa de colocar para a mão direita de acordo com a fileira de reentrâncias.

Na tentativa de retirar:

$1=$ início da tentativa de retirar para a mão direita,

$2=$ meio da tentativa de retirar para a mão direita e
$3=$ final da tentativa de retirar para a mão direita de acordo com a fileira de reentrâncias.

Os orifícios em cinza representam os pinos já depositados em cada reentrância de acordo com a ordem prescrita.

Os orifícios em cinza representam os pinos já depositados em cada reentrância de acordo com a ordem prescrita. 
Para a aquisição da medida de tempo de resposta (Tresp) foi utilizado um cronômetro digital Vollo (Vollo Sports, modelo $\mathrm{n}^{\circ}$ VL1809). Para a orientação e posicionamento dos voluntários, foi utilizado a fita métrica Coats Corrente (Coats Corrente Ltda, marca fita métrica Coats $150 \mathrm{~cm}$ ). A higienização das mãos e dos equipamentos foi realizada utilizando álcool em gel (Rioquimica S.A Industria Farmacêutica, Rialcool álcool etílico $77^{\circ} \mathrm{GL}$ $70^{\circ}$ INPM). Para a proteção individual dos voluntários, foi disponibilizado máscaras descartáveis de proteção facial Descarpack (Descarpack - Efficiency in Disposables, modelo Máscara Cirúrgica Tripla com Elástico).

Para a realização do experimento, foram adotados procedimentos de segurança referentes aos protocolos pandêmicos da COVID19, orientados pela Organização Pan-Americana de Saúde (OPAS) e a Organização Mundial de Saúde (OMS). Os voluntários foram previamente orientados quanto à necessidade de uso da máscara de proteção individual, higienização das mãos e manutenção de medidas de distanciamento previamente e durante a coleta de dados. Todos os equipamentos utilizados na coleta foram higienizados antes e após o início de cada coleta. A realização da coleta aconteceu em local previamente estabelecido entre pesquisador e voluntário, de forma a garantir o cumprimento dos protocolos de segurança (OPAS; OMS 2020).

Os voluntários assinaram o Termo de Consentimento Livre e Esclarecido (TCLE), confirmando o pleno acordo da participação voluntária no estudo e concordando com os testes a serem realizados e procedimentos a serem adotados. Em seguida, os voluntários responderam o Inventário de Dominância Lateral de Edimburgo (OLDFIELD, 1971), utilizado para o cálculo do Índice de Preferência Lateral (TEIXEIRA; PAROLI, 2000).

Após a realização dos procedimentos anteriores, a coleta dos dados foi realizada mediante a aplicação do teste de destreza manual Grooved Pegboard Test. O voluntário foi orientado a sentar-se confortavelmente em uma cadeira, com a linha média do corpo alinhada ao equipamento. Marcas de orientação para o posicionamento corporal, posicionamento das mãos foram colocadas na mesa como forma de garantir que a posição de início e de fim do movimento fosse a mesma para todos os voluntários. Com o auxílio de uma fita métrica foram aferidos sobre a mesa a distância de 60 centímetros entre uma extremidade e outra da fita onde foram distribuídas as marcações afixadas com fita adesiva. Foram utilizados o mesmo tamanho e material para todos os voluntários.

Em relação ao desempenho no teste, o tempo de resposta (Tresp) foi expresso pelo intervalo entre o comando para iniciar o movimento e o fim do movimento. O voluntário iniciou o teste com a mão de execução do movimento em cima da mesa ao lado do equipamento, ao comando "vai" o cronômetro foi disparado e o voluntário deveria iniciar o movimento o mais rápido possível. Ao finalizar o movimento o voluntário retornou a mão para a posição inicial e o cronômetro foi interrompido. Para a aquisição da medida foi utilizado um cronometro digital, com o tempo aferido em segundos.

Todos os voluntários receberam instrução verbal da tarefa de forma padronizada. Para a familiarização com o teste, os voluntários realizaram o encaixe e a retirada dos pinos dos orifícios referentes às duas primeiras fileiras de reentrâncias, de acordo com a ordem prescrita para cada mão, apenas uma vez. Cada voluntário realizou duas tentativas de colocar e duas tentativas de retirar com cada uma das mãos de forma alternada com a mão inicial e secundária, respeitando um intervalo de dois minutos entre as tentativas e obedecendo a ordem de contrabalanceamento entre as mãos e os voluntários. Inicialmente os voluntários colocaram os pinos e em seguida retiraram. O tempo aferido em cada tentativa expressou o desempenho individual de cada voluntário no uso da respectiva mão.

Os dados foram analisados quanto à normalidade através do teste de Shapiro-Wilk que indicou distribuição normal $(p>0,05)$. Os dados foram transformados de segundos para milissegundos (ms), e, em seguida foram calculadas as médias do tempo de resposta entre a primeira e a segunda execução com cada mão para as tarefas de colocar e retirar os pinos. Para comparar o desempenho das mãos foram realizados testes $t$ para amostras dependentes.

Para calcular o índice de assimetria (IA) para os destros e canhotos, foram utilizadas as seguintes equações (TEIXEIRA; PAROLI, 2000; FERNANDES et al., 2016):

$$
\begin{aligned}
& I A=(D-E / D+E) \times 100 \text { (destros) } \\
& I A=(E-D / E+D) \times 100 \text { (canhotos) }
\end{aligned}
$$

Onde $\mathrm{QL}=$ índice de assimetria, $\mathrm{D}=$ valores para mão direita, $\mathrm{E}=$ valores para mão esquerda.

Para comparação dos índices de assimetria entre os grupos foi utilizado um teste t para amostras independentes. $O$ valor de significância adotado foi de $\alpha=5 \%$.

\section{RESULTADOS E DISCUSSÃO}

\section{Preferência lateral}

A preferência lateral foi calculada a partir das respostas obtido no Inventário de Dominância Lateral de Edimburgo (OLDFIELD, 1971). Os resultados podem ser observados na Tabela $1 \mathrm{e}$ mostram média de $97 \%$ no índice de preferência manual direita em indivíduos destros. Para os indivíduos canhotos a média do índice de preferência manual esquerda apresentada foi de $91 \%$.

Tabela 1. Resultados do Inventário de Dominância Lateral de Edimburgo (OLDFIELD, 1971).

\begin{tabular}{ccc}
\hline \multirow{2}{*}{ Voluntário } & \multicolumn{2}{c}{ Quociente de lateralidade } \\
\cline { 2 - 3 } & Destros (\%) & Canhotos (\%) \\
\hline 1 & $100 \%$ & $85 \%$ \\
2 & $95 \%$ & $95 \%$ \\
4 & $100 \%$ & $80 \%$ \\
5 & $100 \%$ & $100 \%$ \\
6 & $85 \%$ & $80 \%$ \\
7 & $100 \%$ & $100 \%$ \\
8 & $95 \%$ & $95 \%$ \\
9 & $100 \%$ & $100 \%$ \\
10 & $100 \%$ & $85 \%$ \\
Média & $95 \%$ & $90 \%$ \\
\hline
\end{tabular}




\section{Desempenho das mãos - tarefa de colocar os pinos}

$\mathrm{Na}$ condição de colocar os pinos o teste t detectou diferenças significativas entre as mãos direita e esquerda dos destros $[t(d f=9)=-7,16 ; p=0,01]$ e canhotos $[t(d f=9)=4,80 ; p=0,01]$. Para os destros a mão direita apresentou menor tempo de resposta comparada a mão esquerda. Por outro lado, para os canhotos o resultado foi inverso, este grupo apresentou melhor desempenho para a mão esquerda quando comparada com a direita (Figura 3a).

\section{Desempenho das mãos - tarefa de retirar os pinos}

Para a condição de retirar os pinos o teste $t$ não detectou diferença significante entre as mãos direita e esquerda para os destros $[t(d f=9)=-1,96 ; p=0,08]$ e nem para os canhotos $[t(d f=9)=1,27 ; p=0,23]$ (Figura $3 b$ ).
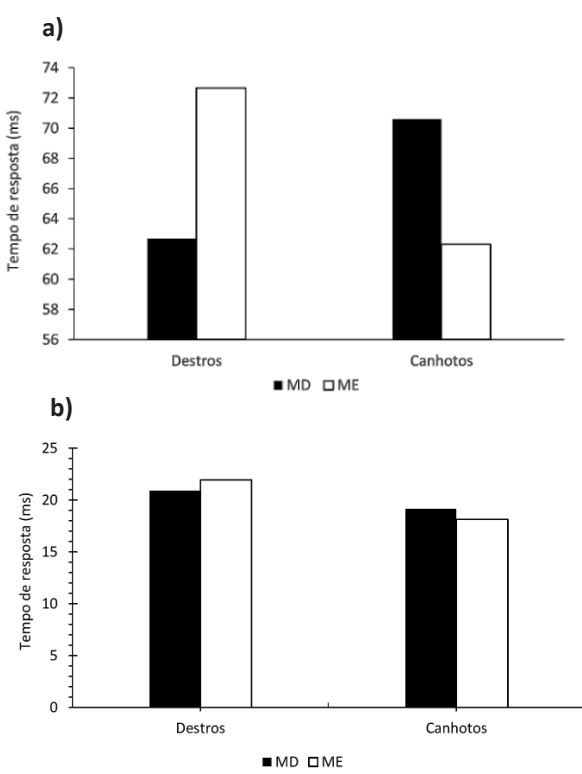

Figura 3. Tempo de resposta da tarefa de colocar os pinos (a); Tempo de resposta durante a tarefa de retirar os pinos (b).

\section{Índice de assimetria destros $\mathrm{x}$ canhotos}

$\mathrm{Na}$ análise do índice de assimetria entre os grupos o teste $t$ não detectou diferenças significativas para as tarefas de colocar os pinos $[t(d f=18)=-0,76 ; p=0,71]$ e retirar os pinos $[t(d f=18)=0,42 ; p=0,14]$ (Figura 4).

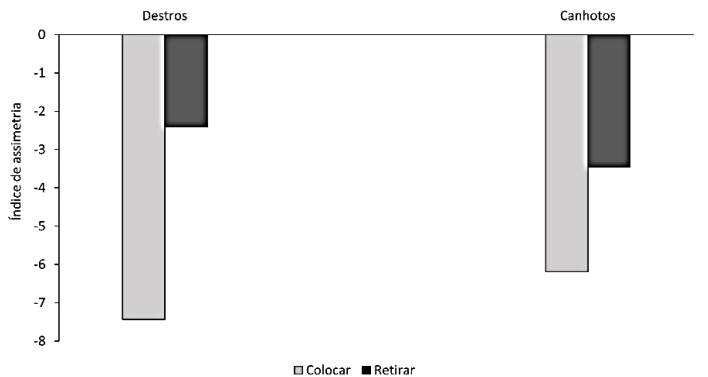

Figura 4. Comparação do índice de assimetria entre os grupos destros e canhotos.
Através da análise de desempenho de uma tarefa de destreza manual, buscou-se analisar as diferenças no desempenho das mãos preferidas e não preferidas entre indivíduos de mesma população e a assimetria de desempenho entre destros e canhotos.

Quanto à lateralidade, o resultado do índice de dominância lateral apresentado no estudo indicou que a média em indivíduos destros foi de $97 \%$, e em indivíduos canhotos $91 \%$, o que corrobora o fato de que indivíduos canhotos tendem a ser menos lateralizados em relação a destros como nos estudos de Bagi et al. (2011) e Steenhuis et al. (1999).

Durante a tarefa de colocar os pinos, os resultados mostram uma diferença significativa no desempenho entre a mão preferida e não preferida para os indivíduos destros e canhotos. Os resultados convergem para o entendimento de que, tanto para destros quanto para os canhotos a mão preferida tende a apresentar melhor desempenho quando comparada ao uso da mão não preferida, estes resultados concordam com os estudos de Salvador et al. (2017) e Fernandes et al. (2018). Os resultados do presente estudo mostram que em canhotos existe um comportamento similar no desempenho com o uso da mão preferida em relação a mão não preferida, sendo que a mão preferida assim como em destros apresentou melhor desempenho em relação a mão contralateral. Essa preferência se trata de voluntários autodeclarados e certificados pelos resultados do Inventário Lateral de Edimburgo (OLDFIELD, 1971).

Na condição de retirar os pinos, os resultados não mostram diferenças significativas no desempenho entre a mão preferida e não preferida, tanto para destros quanto para canhotos, levando ao entendimento de que para esta tarefa, a diferença no desempenho entre as mãos se manteve equilibrada em destros e em canhotos. Esse resultado contrapõe o pressuposto que, devido ao fato de indivíduos canhotos serem menos lateralizados, estes apresentariam uma menor diferença no desempenho entre as mãos quando comparados aos destros (GORYNIA; EGENTER, 2000). Esse fato pode ser decorrente do tipo de tarefa desempenhada já que a diferença no desempenho das mãos nas mais diversas tarefas tem sido conferida a uma proficiência relativa dos hemisférios cerebrais no processamento de informações percepto-motoras (TODOR; SMILEY, 1985), sendo necessário a aplicação de outros testes para a análise das diferenças no desempenho das mãos.

No presente estudo os resultados não apresentaram diferença significante entre os índices de assimetria de desempenho dos grupos de destros e canhotos, apesar de indivíduos canhotos apresentarem menor índice na tarefa de colocar os pinos, e os destros apresentarem menor índice para a tarefa de retirar os pinos, estes resultados não representaram relevância estatística na comparação da assimetria de desempenho nas tarefas colocar e retirar. Os resultados do estudo de Fernandes et al. (2017) corroboram os achados do presente estudo no que diz respeito à ausência de diferenças entre o índice de assimetria. Ademais, para ambos os grupos, destros e canhotos, foi verificado um menor índice de assimetria na tarefa de retirar os pinos em comparação à tarefa de colocar os pinos.

Estes achados sugerem que as assimetrias entre os grupos se destacam em tarefas de maior complexidade em comparação com tarefas com menor complexidade, o que já foi reportado previamente na literatura (BRYDEN et al., 2007). De fato, 
a tarefa de colocação dos pinos requer dos participantes uma maior demanda visual previamente ao movimento de preensão do pino, em função da necessidade de ajuste da orientação final das reentrâncias nos orifícios, evidenciando uma maior demanda cognitiva e por ajustes motores finos. Isso evidencia não apenas a demanda por controle de força na preensão do pino, mas também de ajuste da orientação espacial ao longo do transporte do pino, indicando a existência de mais um componente da tarefa. Por sua vez, na tarefa de retirada dos pinos a demanda visual prévia à preensão dos pinos é menor, uma vez que os pinos já se encontram na posição correta desde o início, e não existe a necessidade de realizar ajustes finos durante o transporte do pino ao destino final, elucidando maior demanda energética. Assim, o maior nível de complexidade na tarefa de colocar pinos, que implica em um maior nível de desafio aos indivíduos, pode ter contribuído para a observância dos maiores índices de assimetria.

Era esperado que os indivíduos canhotos, pelo fato de possuírem um maior equilíbrio na utilização de ambas as mãos, apresentassem uma menor diferença de desempenho das mãos, verificada através do índice de assimetria. De forma geral, os resultados encontrados no presente estudo confirmam parcialmente a hipótese sugerida de que indivíduos com lateralidade distinta possivelmente apresentam diferenças no desempenho de uma tarefa de destreza manual. Por um lado, observamos diferenças no desempenho das mãos entre os grupos, mostrando a especificidade do comportamento motor dos indivíduos com lateralidade distinta. Por outro lado, não podemos afirmar que os indivíduos canhotos, por serem menos lateralizados, são menos assimétricos.

É importante ponderar que, na investigação das assimetrias manuais de desempenho é fundamental tratar a especificidade da tarefa e suas demandas. Nesse contexto, para uma tarefa de destreza manual o desempenho das mãos de indivíduos destros e canhotos é distinto, entretanto, as diferenças no desempenho das mãos intragrupo não se diferem significativamente na realização dessa tarefa.

O estudo possui um número de participantes restrito e engloba questões relacionadas à lateralidade e controle motor fino. A lateralidade deve ser entendida como um elemento dinâmico no comportamento humano, portanto, os resultados do estudo precisam ser utilizados com cautela podendo ser generalizados apenas para tarefas com as mesmas demandas em termos de controle motor fino. Dessa forma, estudos futuros podem ampliar as investigações sobre as questões discutidas no presente estudo a partir de um número amostral maior e com a utilização de diferentes perspectivas de indagação, como exemplo, faixas etárias distintas, análise das diferenças entre os sexos no controle manual, assim como análise em tarefas com diferentes demandas.

\section{CONCLUSÃO}

Em síntese, no presente estudo foi possível observar que para os indivíduos com lateralidade distinta, o desempenho nas tarefas de destreza manual com o Grooved Pegboard Test, apresentou diferenças significativas para o desempenho com a mão preferida tanto para destros quanto para canhotos na tarefa de colocar os pinos, onde a mão preferida apresentou melhor de- sempenho. Já para a tarefa de retirar os pinos não houve diferenças estatísticas significante tanto para destros quanto para canhotos. Referente à assimetria de desempenho intergrupo não foram observadas diferenças significantes, entretanto, ambos os grupos apresentaram maior índice de assimetria para a tarefa de colocar os pinos, ou seja, tarefa em que há maior demanda cognitiva em função de sua maior complexidade.

A compreensão das diferenças no desempenho entre as mãos em tarefas manuais demonstra grande complexidade de ilustração entre destros e canhotos, visto que distintas tarefas e diferentes manifestações de preferência lateral influenciam no desempenho com a mão preferida e não preferida. A continuidade do estudo referente ao desempenho das mãos se faz necessário a fim de elucubrar o desempenho em indivíduos com lateralidade distinta e evidenciar a realidade do desempenho das mãos e as possíveis assimetrias entre indivíduos destros e canhotos. O desdobramento na compreensão do processamento de informações percepto-motoras e da especialização hemisférica pode indicar caminhos para compreensão do desempenho manual.

\section{REFERÊNCIAS}

ANNETT, M.; KILSHAW, D. Right-and left-hand skill II: Estimating the parameters of the distribution of I-r differences in males and females. British Journal of Psychology, London, v. 74, n. 2, p. 269-83, 1983.

BAGI, J. G.; KUDACHI, P. S.; GOUDAR, S. S. Influence of motor task on handedness. Al Ameen Journal of Medical Sciences, Bangalore, v. 4, n. 1, p. 87-91, 2011.

BOULINGUEZ, P.; BARTHÉLÉMY, S.; DEBU, B. Influence of the movement parameter to be controlle don manual RT asymmetries in right-handers. Brain and Cognition, San Diego, v. 44, n. 3, p. 653-61, 2000.

BRYDEN, P. J.; ROY, E. A. A new method of administering the Grooved Pegboard Test: Performance as a function of handedness and sex. Brain and Cognition, San Diego, v. 58, n. 3, p. 258-68, 2005.

BRYDEN, P. J.; ROY, E. A.; ROHR, L. E.; EGILO, S. Task demands affect manual asymmetries in pegboard performance. Laterality, London, v. 12, n. 4, p. 364-77, 2007.

DE OLIVEIRA, T. F. BARAVIEIRA, E., BEGGITATO, A., ALVES, V. H. Preferência lateral percebida e diagnosticada em adolescentes. Revista Brasileira de Ciências do Esporte, São Paulo, v. 38, n. 4, p. 315-20, 2016.

DE SOUZA, R. M.; TEIXEIRA, L. A. Sobre a relação entre filogenia e ontogenia no desenvolvimento da lateralidade na infância. Psicologia: Reflexão e Crítica, Porto Alegre, v. 24, n. 1, p. 62-70, 2011.

DESROSIERS, J. ROCHETTE, A., HÉBERT, R., BRAVO, G., The Minnesota Manual Dexterity Test: Reliability, validity and reference values studies with healthy elderly people. Canadian Journal of Occupational Therapy, New York, v. 64 , n. 5, p. 270-6, 1997.

ELLIOTT, D.; ROY, E. A. Manual asymmetries in motor performance. CRC Press: Florida, 1996.

FERNANDES, L. A.; APOLINÁRIO-SOUZA, T.; SOUZA, B. C. G. S.; SALLES, I. Análise da assimetria manual na performance do Grooved Pegboard Test utilizando um acelerômetro. Brazilian Journal of Motor Behavior, Porto Alegre, v. 12 , n. 1, p. 1-11, 2018.

FERNANDES, L. A.; APOLINÁRIO-SOUZA, T; OLIVEIRA, J. R. V; OLIVEIRA, S. R.; LAGE, G. M. Aplicação da Estimulação Transcraniana por Corrente Contínua na melhoria do desempenho manual. Revista Portuguesa de Ciências do Desporto, Porto, v. 3, n. S3A, p. 13-24, 2017.

FERNANDES, L. A.; SILVA, A.; AUGUSTO, V. M. E.; NOGUEIRA, N. G. H. M.; FERREIRA, B. P; JUNQUEIRA, C.; LAGE, G. M. Analysis of laterality and manual dexterity in children with autistic spectrum disorder. Revista Brasileira de Educação Especial, Bauru, v. 26, n. 4, p. 453-68, 2020.

FERNANDES, L., A. Os efeitos da modulação hemisférica nas assimetrias manuais: inibição inter-hemisférica e aumento da excitabilidade hemisférica. 2016. 119f. Tese (Doutorado em Ciências do Esporte) - Universidade Federal de Minas Gerais, Belo Horizonte, 2016. 
FUELSCHER, I.; HYDE, C.; EFRON, D.; SILK, T. J. Manual dexterity in late chilhood is associated with maturation of the corticospinal tract. Neurolmage, Orlando, v. 226, p. 1-10, 2021.

GIAGAZOGLOU, P., FOTIADOU, E., ANGELOPOULOU, N., TSIKOULAS, J., TSIMARAS, V. Gross and fine motor skills of left-handed pre school children. Perceptual and Motor Skills, Missoula, v. 92, n. 3, p. 1122-8, 2001

GOODALE, M. A. Brain asymmetries in the control of reaching: Vision and action. Amsterdam: North-holland, 1990.

GORYNIA, I.; EGENTER, D. Intermanual coordination in relation to handedness: Familial sinistrality and lateral preferences. Córtex, Milano, v. 36, n. 1, p. 1-18, 2000.

GURD, J. M., SCHULZ, J., CHERKAS, L., EBERS. G. C. Hand preference and performance in 20 pairs of monozygotict wins with discordant handedness. Córtex, Milano, v. 42, n. 6, p. 934-45, 2006.

JUDGE, J.; STIRLING, J. Fine motor skill performance in left- andright-handers: Evidence of an advantage for left-handers. Laterality, London, v. 8, n. 4, p. 297-306, 2003.

MARCONI, A. J.; MONTEIRO, P. H.; OKAZAKI, V. H. A. Changing handedness: What can we learn from preference shift studies? Neuroscience and Biobehavioral Reviwes, Oxford, v. 107, p. 313-9, 2019.

McGRATH, R. L.; KANTAK, S. S. Reduced asymmetry in motor skill learning in left-handed compared to right-handed individuals. Human Movement Science, Amsterdan, v. 45, p. 130-41, 2016

OLDFIELD, R. C. The assessment and analysis of handedness: The Edinburgh inventory. Neuropsychologia, Oxford, v. 9, n. 1, p. 97-113, 1971.

OPAS. Organização Pan-Americana de Saúde. Paho. OPAS, c2020. Disponível em: <https://www.paho.org/pt/covid19>. Acessado em: 25 de setembro de 2020.

PETERS, M.; PORAC, C.; COREN, S. Lateral preferences and human behavior. The American Journal of Psychology, Champaign, v. 96, n. 2, p. 296-8, 1983.
PRZYBYLA, A · GOOD D. C. SAINBURG, R. S. Dynamic dominance varies with handedness: reduced interlimb asymmetries in left-handers. Experimental Brain Research, Amsterdam, v. 216, n. 3, p. 419-31, 2012.

SALVADOR, M. G.; UGRINOWITSCH, H.; ROMANO, M. A.; MARQUES, D.; APOLINÁIO-SOUZA, T.; MENEZES, G. Transcranial direct current stimulation and manual asymmetries: The effect of the stimulation on the manual dexterity. Journal of Physical Education, Maringá, v. 28, n. 1, p. 3-5, 2017.

SERAFIN, G.; PERES, L. S.; CORSEUIL, H. X. Lateralidade: conhecimentos básicos e fatores de dominância em escolares de 7 a 10 anos. Caderno de Educação Física, Marechal Cândido Rondon, v. 2, n. 1, p. 11-30, 2000.

STEENHUIS, R. E.; BRYDEN, M. P. The relation between hand preference and hand performance: what you get depends on what you measure. Laterality, London, v. 4, n. 1, p. 3-26, 1999.

TEIXEIRA, L. A. Controle motor. São Paulo: Manole, 2006.

TEIXEIRA, L. A.; PAROLI, R. Assimetrias Laterais em Ações Motoras: Preferência Versus Desempenho. Motriz, Rio Claro, v. 6, n. 1, p. 1-8, 2000.

TODOR, J.I.; SMYLE, A. Manual asymmetries in control motor. Neuropsychological studies of apraxia and related disorders. Amsterdam: North Holland, 1985.

TZOURIO-MAZOYER, N.; LABACHE, L.; ZAGO, L.; HESLING, I.; MAZOYER, B. Neural support of manual preference revealed by BOLD variations during right and left finger-tapping in a sample of 287 healthy adults balanced for handedness. Laterality, London, v. 6, p. 1-23, 2021

VAN DE VEN-STEVENS, L. A. MUNNEKE, M.; TERWEE, C. B. SPAUWEN, P. H. VAN DER LINDE, $\mathrm{H}$. Clinimetric properties of instruments to assess activities in patients with hand injury: a systematic review of the literature. Archive of Physical Medicine and Rehabilitation, Philadelphia, v. 90, n. 1, p. 151-69, 2009

WOODWORTH, R. S. The accuracy of voluntary movement. Journal of Nervous and Mental Disease, Philadelphia, v. 26, n. 12, p. 743-52, 1899.

\section{CONFLITO DE INTERESSE}

Os autores do estudo declaram não haver conflito de interesses.

\section{FINANCIAMENTO}

Este estudo não teve apoio financeiro.

\section{ORCID E E-MAIL DOS AUTORES}

Lidiane Aparecida Fernandes (Autor Correspondente) ORCID: 0000-0001-8909-1612.

E-mail: lidianefernandes12@yahoo.com.br

Wallace Tadeu Alves

ORCID: 0000-0003-0206-1352.

E-mail: wallacehd@live.com

Simara Regina de Oliveira Ribeiro

ORCID: 0000-0003-4330-5261.

E-mail: simararibeiro@gmail.com

Lucas Savassi Figueiredo

ORCID: 0000-0001-5853-4978.

E-mail: savassi88@gmail.com

Natália Fontes Alves Ambrósio

ORCID: 0000-0002-0553-9852.

E-mail: nataliambrosio@gmail.com

Crislaine Rangel Couto

ORCID: 0000-0003-3305-3208.

E-mail: crislainerangel@hotmail.com

Guilherme Menezes Lage

ORCID: 0000-0001-8296-3676.

E-mail: menezeslage@gmail.com 